\title{
An Electrodynamic/Magnetorheological Clutch Powered by Permanent Magnets
}

\author{
R. Rizzo, A. Musolino, and H.C. Lai
}

\begin{abstract}
This paper presents an electrodynamic/magnetorheological fluid-based clutch activated using permanent magnets attached to one shaft. During the electromechanical transient phase, when the two clutch shafts rotate at different speed, the Permanent Magnets (PMs) induce eddy currents on some conductive materials strategically placed in the system. The interaction between these currents and the magnetic induction produces an electromagnetic torque which is added to that of the Magneto-Rheological Fluid (MRF), helping the clutch engagement in the start up phase when an extra torque is necessary to overcome possible static frictions. Once the two shafts have reached the same speed, the eddy currents (and consequently the electromagnetic torque) vanish and the torque transmission is assured by the MRF only. The conductive material is arranged as a copper sheet and the performance of the device is investigated. The proposed clutch is analyzed using a 3-D finite-element code which takes into account the B-H curve of the nonlinear materials (e.g. MRF, Permanent Magnets and ferromagnetic materials) and the movement of the different parts. Finally, in order to verify the FEM model, some experimental measurements are performed on a prototype.
\end{abstract}

Index Terms-Magneto-Rheological Clutch, Permanent Magnets, FEM, Experimental measurements, Electrodynamic torque.

\section{INTRODUCTION}

In previous papers [1],[2], the authors presented a fail-safe magnetorheological fluid-based clutch [3],[4],[5],[6], excited by permanent magnets and operating without electrical power. It was designed by using a magnetic 3-D Finite Elements code [7] and a magneto/mechanical model [1], developed for the purpose and capable of exploiting the magnetic mesh in order to obtain the transmissible torque. As a result a preliminary prototype [8], shown in figure 1 , was built and some experimental measurements were taken in order to both verify the effective operation of the proposed system and validate the magneto/mechanical FEM model.

However, in this paper a new solution is presented. It exploits the "eddy currents" induced on a conductive region by the relative rotation of the PMs around their axis (motional effects). In this way an electromagnetic torque is produced and added to the torque transmitted by the magnetorheological fluid (MRF), thus increasing the clutch performance.

The paper is organized as follows. Section II briefly reviews the characteristics of the previously developed device; Section III presents the newly proposed solution; Section IV describes

A. Musolino and R. Rizzo are with the Department of Energy and Systems Engineering, University of Pisa, Largo Lucio Lazzarino, 56122, Pisa, Italy; e-mail: \{antonino.musolino, rocco.rizzo\} @unipi.it; H.C. Lai is with the Bathwick Electrical Design Limited, unit 15, Brassmill Enterprise Centre, Brassmill Lane, Bath (UK), BA1 3JN

Manuscript received xxxxx; revised yyyyy.

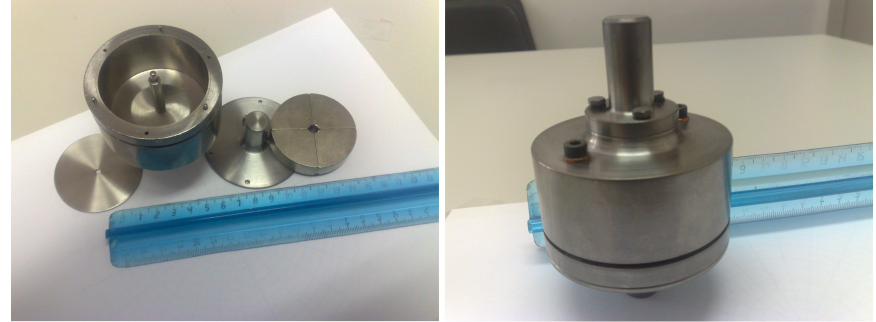

Fig. 1. The MR clutch prototype

the electro-dynamic FEM model with the simulations results; finally, Section V describes the prototype and discusses the results obtained by the comparison between the simulated and measured data.

\section{DeVICE CHARACTERISTICS: SYNTHESIS}

Refer to the cited papers ([1],[2]) for a detailed description of this MRF-based clutch and its operation. For the sake of subsequent clarity, here follows the main characteristics are described. As shown in Fig. 2, the main device components are the primary and secondary shaft, the excitation permanent magnets (PMs) system, and the magnetorheological fluid [9],[10] (MRF140CG produced by Lord Corporation, Cary NC, USA [11]). As for the device materials, the primary shaft dark blue piece and secondary shaft dark green piece are made of ferromagnetic iron AISI-1018. The other parts are made of non-ferromagnetic iron AISI-316L (shaded white and light blue). The excitation system is composed of four $\mathrm{NdFeB} \mathrm{per-}$ manent magnet $90^{\circ}$-poles, alternately magnetized along their diameter direction and forced to move inside the "chamber" (the secondary shaft dashed white part). The linear movement of the permanent magnets along their axial direction allows the device to be controlled [12] [1]: when the PMs are in position 1 (see Figs. 2(a) and 3(a)), the fluid is excited and the clutch transmits a torque of about $3 \mathrm{Nm}$; on the contrary, when the PMs are in position 0 (see figures 2(b) and 3(b)), the fluid is not excited and the transmitted torque $(\simeq 0.2$ to $0.3 \mathrm{Nm})$ is due only to the parasitic frictions (bearings, oil seals, etc.) and to the fluid's natural viscosity. The clutch engagement phase (moving the PMs from position 0 to position 1) is assured by a preloaded spring; the disengagement phase (moving the PMs from position 1 to position 0 ) can be obtained by using an external pneumatic actuator (not shown in figure).

\section{A NEW ADVANCED CONFIGURATION}

In order to increase the transmissible torque, a new configuration can be envisaged. It can be designed by substituting the non-ferromagnetic part of the primary shaft (the 


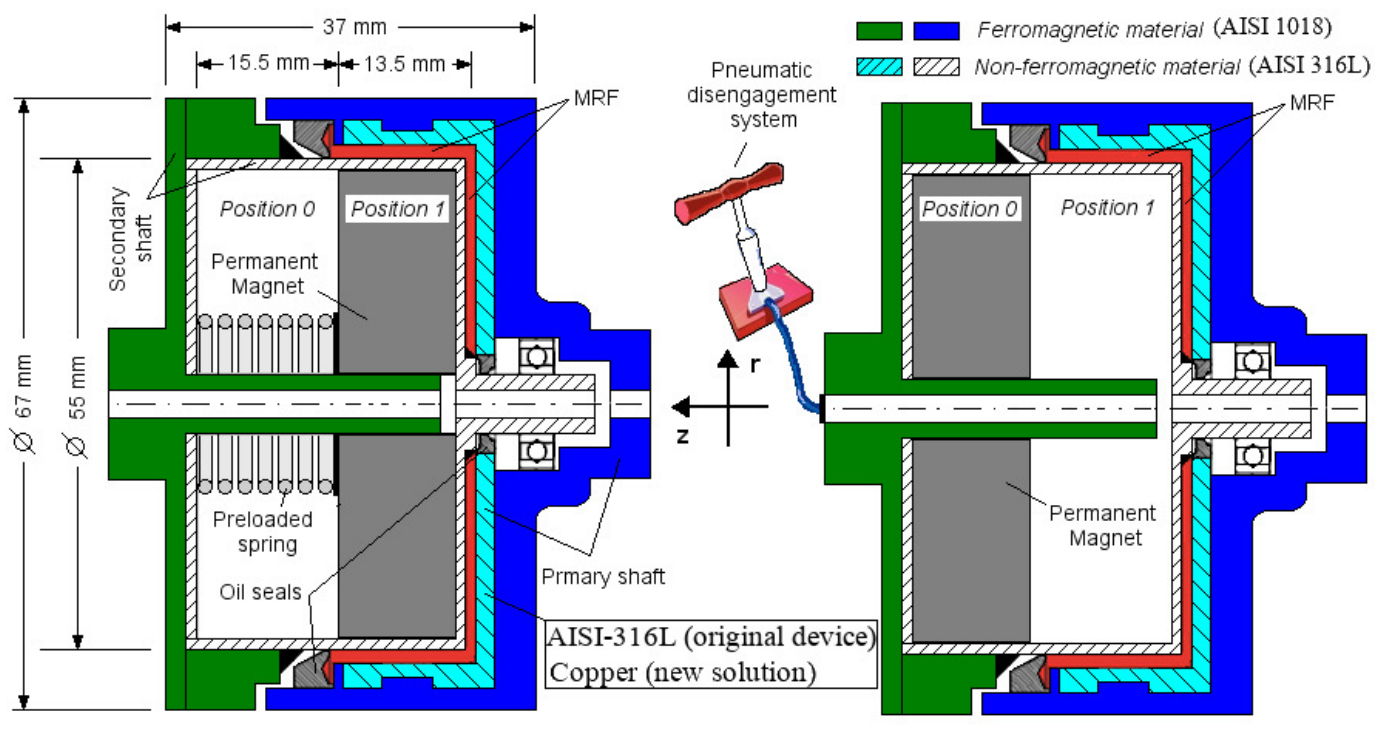

a)

b)

Fig. 2. The schematic view of the device and its operation principle: (a) engaged condition (ON state); (b) disengaged condition (OFF state)
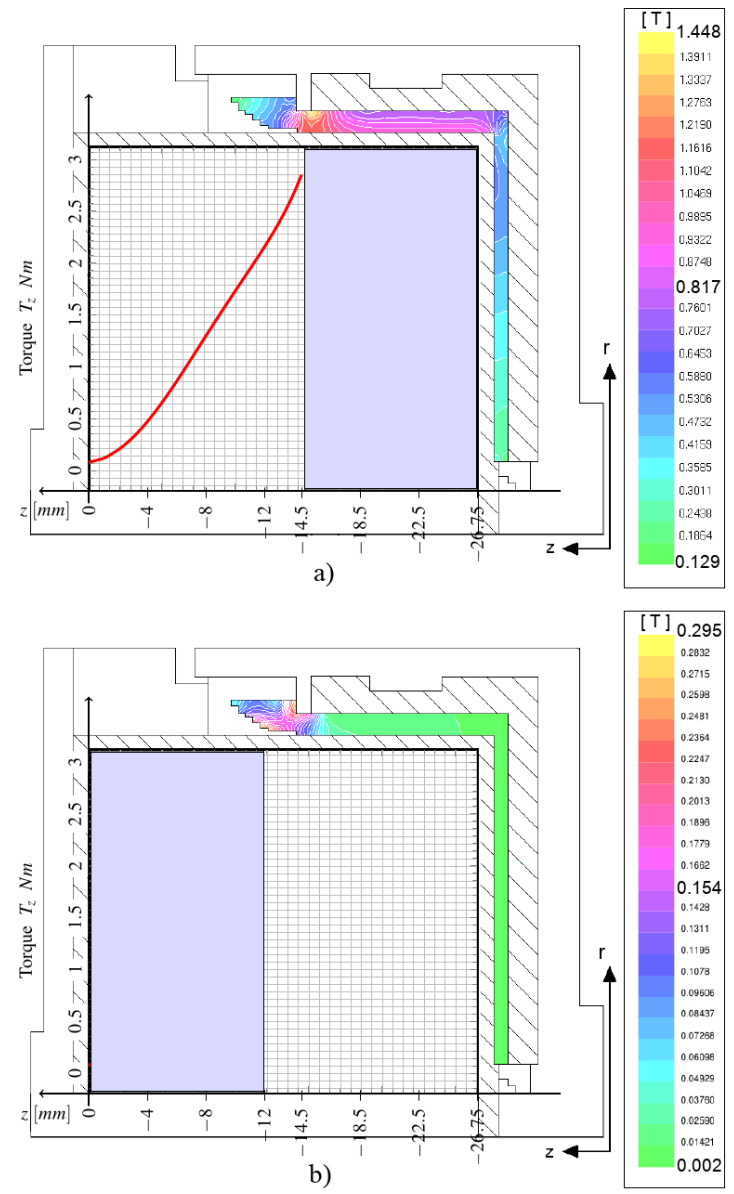

Fig. 3. Torque vs PMs displacement and map of the magnetic induction $B$ inside the MRF: (a) position 1 , clutch engaged, $T_{z} \simeq 3 \mathrm{~N} \mathrm{~m}$; (b) position 0 , clutch disengaged, $T_{z} \simeq 0.2 \div 0.3 \mathrm{~N} \mathrm{~m}$; light blue region) with a copper sheet (see Fig. 2). During the electro-mechanical transient phase, when the two shafts rotate at different speed, the permanent magnets induce eddy currents on such conductive material [13],[14]. This produces an electromagnetic torque which adds to that of the MRF, helping the clutch engagement in the start up phase when an extra torque is necessary to overcome possible static frictions. When the relative speed between the clutch shafts is zero, the eddy currents (and consequently the electromagnetic torque) vanish and the torque transmission is assured by the MRF only. With the proposed solution, during the transient phase, the device operates like an induction motor in which the rotating magnetic fields is obtained by the mechanical rotation of the four-pole PM rotor.

The system was analyzed by means of a dynamic FEM code [7]. It is able to take into account the materials nonlinearity [15], [16] (ferromagnetic materials and MR fluid), the permanent magnets behavior, as well as the motional effect due to the PMs rotation around the device axis.

\section{FEM SIMULATIONS}

Fig. 4(a) shows the FE mesh of the whole structure, containing about $2 \times 10^{6}$ elements and nodes, while Figs. 4(b) and 4(c) show a cut inside the FE model and detail of the new advanced configuration.

A preliminary analysis was carried out in order to identify the characteristics of the new proposed solution. Fig. 5 shows the electromagnetic torque as a function of the number of magnetic poles and the copper thickness, for a relative speed of about $1500 \mathrm{rpm}$ between the primary and secondary shaft.

The results show that the maximum (normalized) torque can be obtained by using an excitation system composed of four magnetic poles, and a copper sheet thickness in the range between $1.5 \mathrm{~mm}$ and $2 \mathrm{~mm}$. The choice of four PM $90^{\circ}$-poles, diametrally magnetized, and the thickness of the 


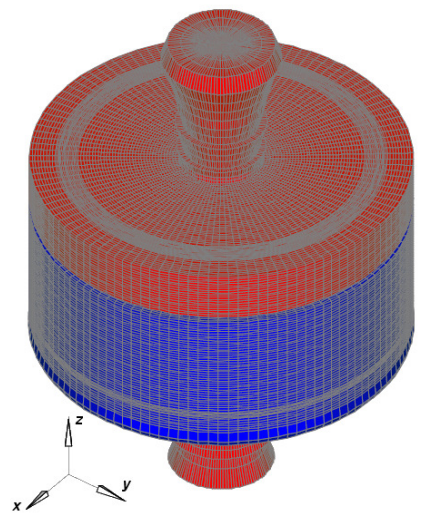

(a) 3D FE model (full device)

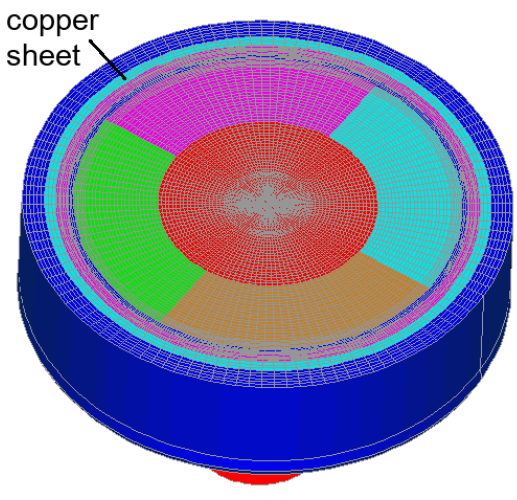

(b) 3D FE model (cut inside)

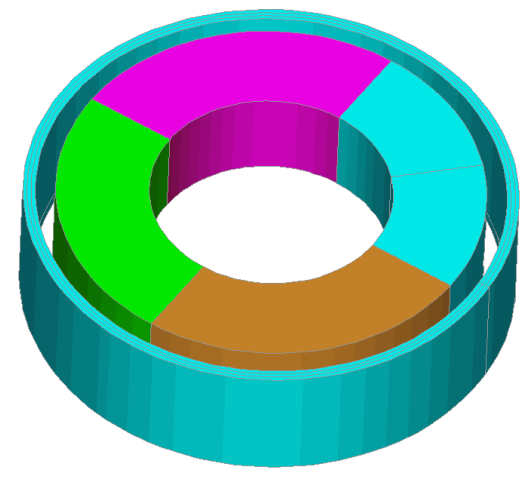

(c) particular (copper sheet and PMs)

Fig. 4. The new configuration with the copper sheet

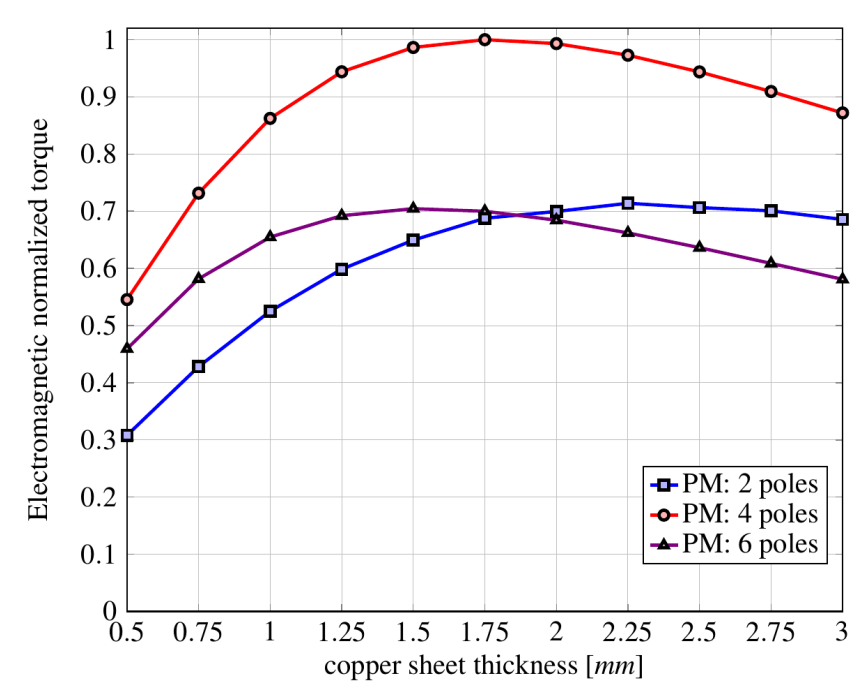

Fig. 5. The (normalized) electromagnetic torque as a function of the number of magnetic poles and the copper sheet thickness (@1500 rpm)

copper sheet of about $2 \mathrm{~mm}$ is the best trade-off between the increase of the torque with frequency (that is with the number of poles of the PMs configuration) and the magnitude of the magnetic flux density [1].

Once the copper sheet was characterized, the performance of the proposed solution was numerically investigated. The simulations were performed imposing on the secondary shaft the speed profile shown in Fig. 6, while keeping the primary shaft blocked. As a consequence, the relative speed between the primary and secondary shaft increases from 0 to $1500 \mathrm{rpm}$ in about $120 \mathrm{~ms}$.

Firstly, the device was analyzed with the MRF removed from the gap. Although this condition does not refer to the real operation of the device, it could be useful to experimentally validate the FEM model. Under this condition, and neglecting the parasitic torque due to the friction, the clutch transmits only the electromagnetic torque due to the eddy currents. Fig. 7 shows the maps of the eddy currents in the sheet when the secondary shaft rotates at

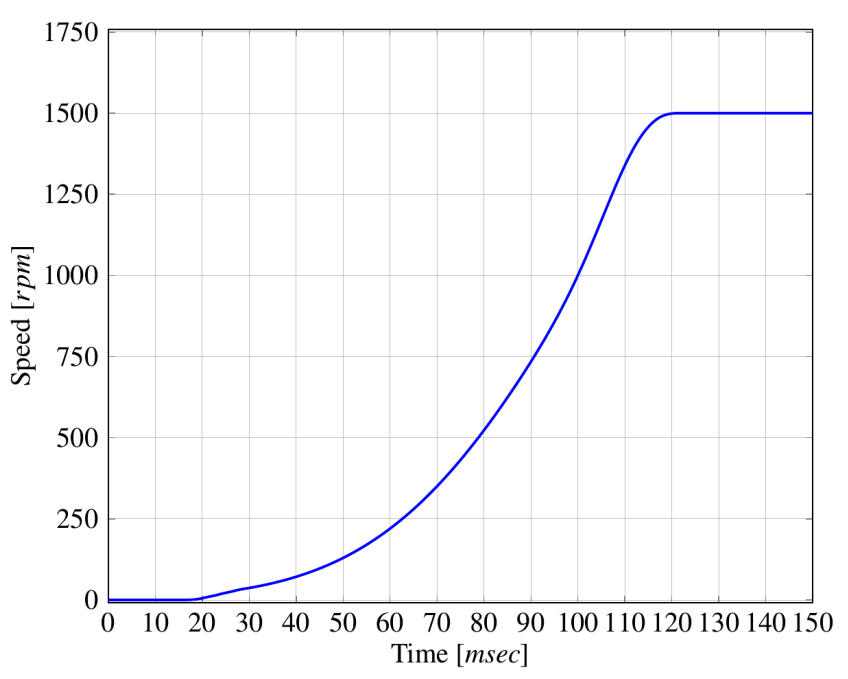

Fig. 6. The relative speed profile between the primary and secondary shaft

a speed of $1500 \mathrm{rpm}$ and the device does not contain the MRF.

A second set of simulations was performed with the gap filled by the MRF. Since the relative magnetic permeability of the fluid is greater than that of the air, its presence modifies the field lines, generally increasing the magnetic field in the whole structure. As a consequence, an increased electromagnetic torque (due to eddy currents only) is expected with respect to the case without the fluid.

Fig. 8 shows the maps of the eddy currents in the sheet in the same speed conditions as the previous figure when the gap is filled by the fluid.

Fig. 9 shows the profile of the torque transmitted by the eddy currents only as a function of the time (that is of the relative speed), with or without the MRF in the gap. The results show that the electromagnetic torque obtained by using the copper sheet is about $0.53 \mathrm{Nm}$ without the MRF, and about $0.8 \mathrm{Nm}$ in the case with the MRF. Accordingly, the presence of the fluid inside the gap increases the electromagnetic torque by about $50 \%$. 


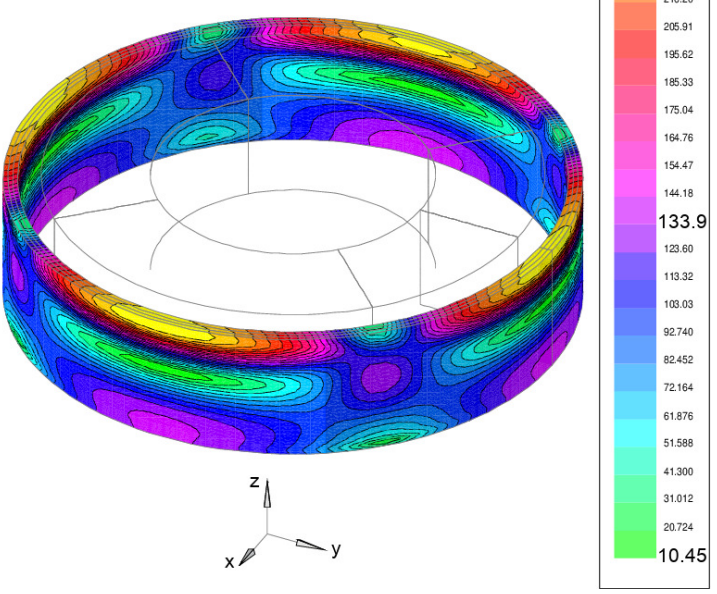

Fig. 7. Eddy Current maps in the copper sheet of the device without MRF.

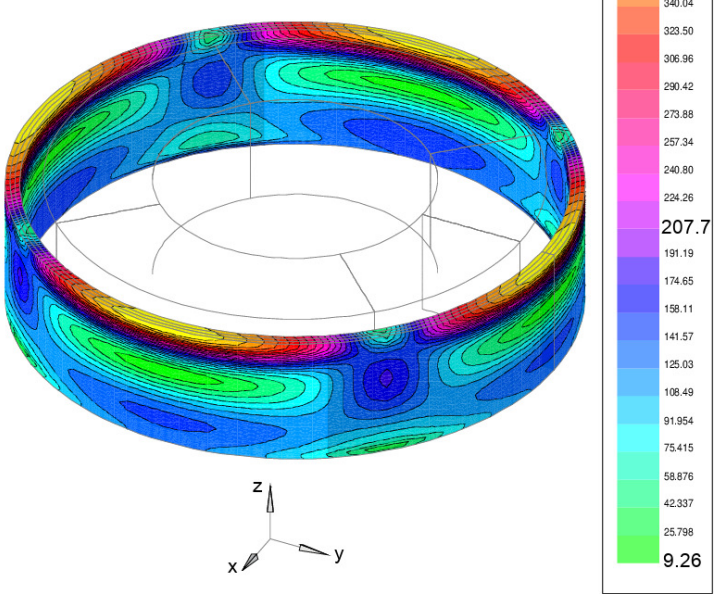

Fig. 8. Eddy Current maps in the copper sheet of the device with MRF.

However, beside the contribution to the torque, the eddy currents affect also the magnetic field inside the fluid, by opposing the source field. In order to evaluate this effect, a deeper numerical investigation was performed. Fig. 10 shows a comparison of the (simulated) magnetic field along a circumference passing through the fluid in the middle of the gap, with and without eddy currents in the copper sheet.

The magnetic field reduction is less than $8 \%$ and generally it occurs in the regions in which the fluid saturates. As a result, the torque transmissible by the MRF only, when eddy currents are taken into account, shows a decrease of about $12 \%$, with respect the torque which can be transmitted without the presence of eddy currents. This value was computed using the accurate magneto/mechanical numerical model described in [1].

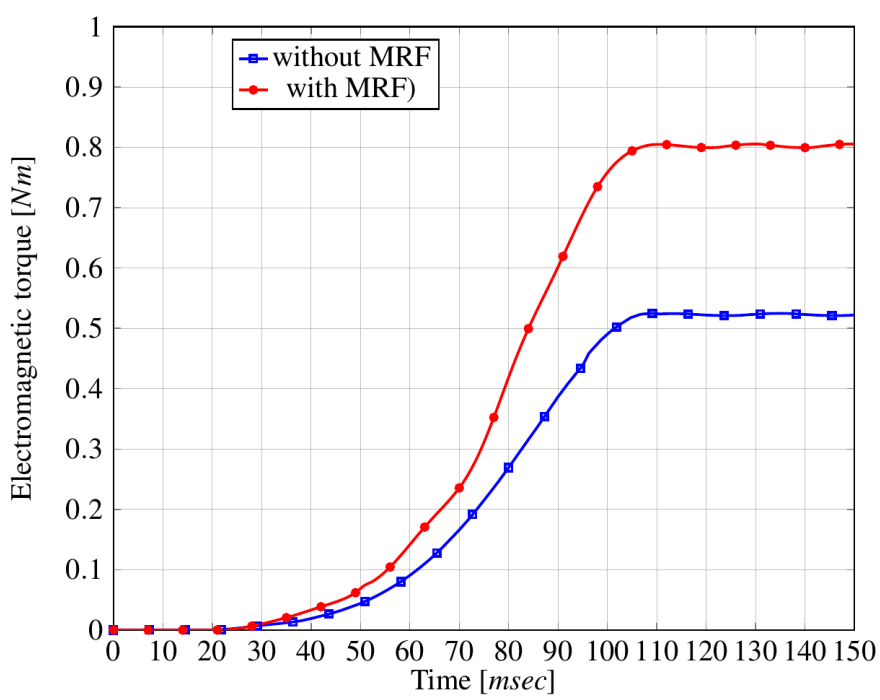

Fig. 9. The electromagnetic torque profile with or without MRF in the gap.

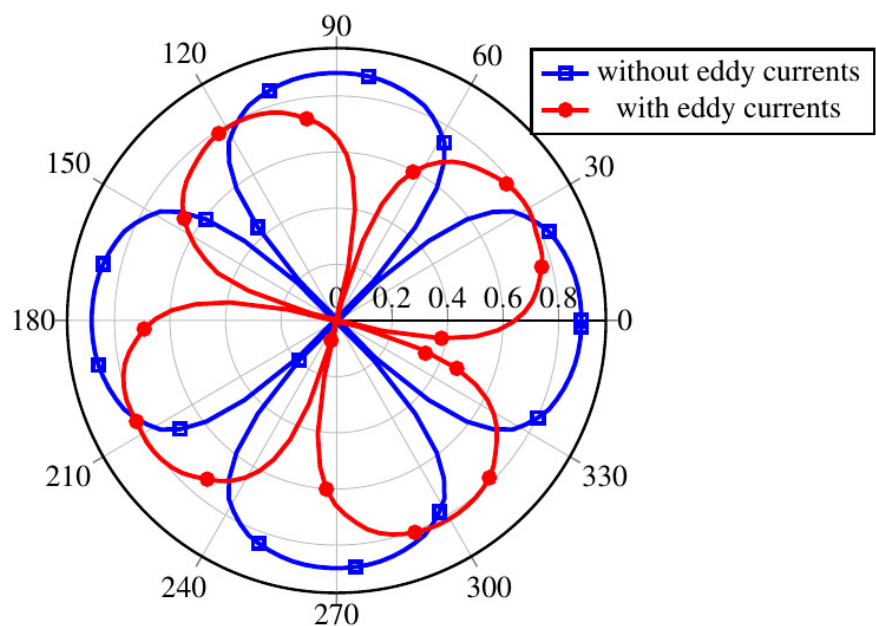

Fig. 10. Comparison between the magnetic flux density $B_{r}$ (in Tesla) along a circumference passing trough the fluid in the middle of the gap, with or without eddy currents in the copper sheet.

\section{EXPERIMENTAL VALIDATION}

On the basis of the results obtained in the previous section, the original prototype, shown in Fig. 1, was modified adding the copper sheet to the primary shaft. Then, by using a test bench shown in Fig. 11, some experimental measurements were performed on the new prototype.

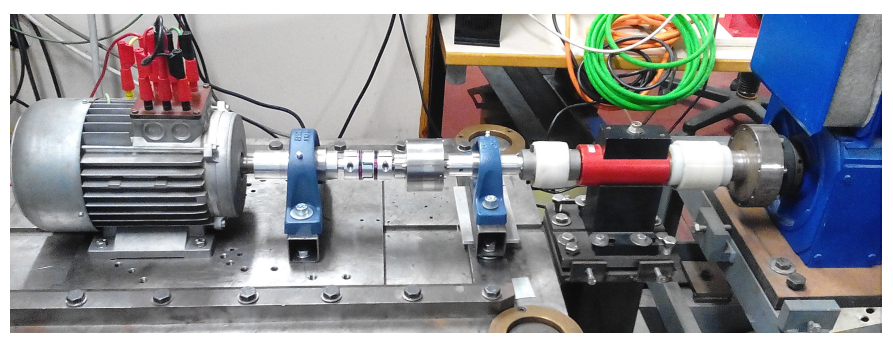

Fig. 11. The test bench 
In order to evaluate the reliability of the dynamic FEM model, the electromagnetic torque due to the copper sheet in the configuration without the MRF was compared with experimental data, as shown in Fig. 12. For the sake of readability, in this figure and in the following ones, a moving average was used to filter the real data and the result (white dashed line) was overlaid on the torque recorded by the instrumentation.

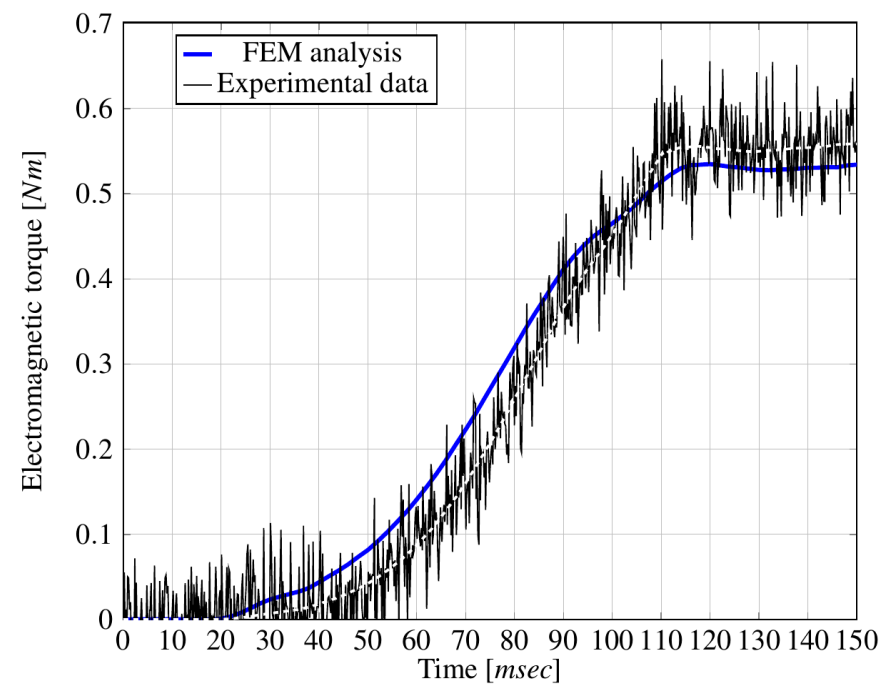

Fig. 12. The electromagnetic torque profile due to copper sheet without MRF comparison between simulation and experimental data.

The analysis shows that the agreement between measured and simulated quantities is satisfactory and therefore the developed FEM model is able to predict the device dynamic operation with acceptable errors.

Although from the numerical point of view it is possible to separate the contributions to the torque due to the eddy currents and to the MRF, the experimental measurement of these two contributions is a quite complex issue. However, in order to estimate these effects, the torque produced by the original prototype was compared with that obtained by the new one (with the copper sheet). Since the ratio between the conductivity of the copper (in the new advanced device) and the conductivity of the AISI-316L (in the original prototype) is about $\sigma_{c u} / \sigma_{A I S I 316 L}=5.8 \times 10^{7} / 1.4 \times 10^{6} \simeq 40$, the eddy currents in the previously developed clutch can be reasonably neglected.

The tests were performed as follows. The secondary shaft, with the PMs in the engaged position, was driven imposing the smoothed (relative) speed profile of Fig. 6, while the primary shaft was kept at rest $\left(\Omega_{1}=0\right)$.

The results are shown in figure 13. The Profile A refers to the torque obtained by the original prototype, in which the eddy currents are negligible. However, as described in [2], the torque transmitted by the MRF increases as a function of the relative speed between the two shafts due to the viscous and friction components of the torque.

The Profile B concerns the new advanced prototype. Considering that the tests for both the prototypes were performed under the same conditions, the different values of torque can be

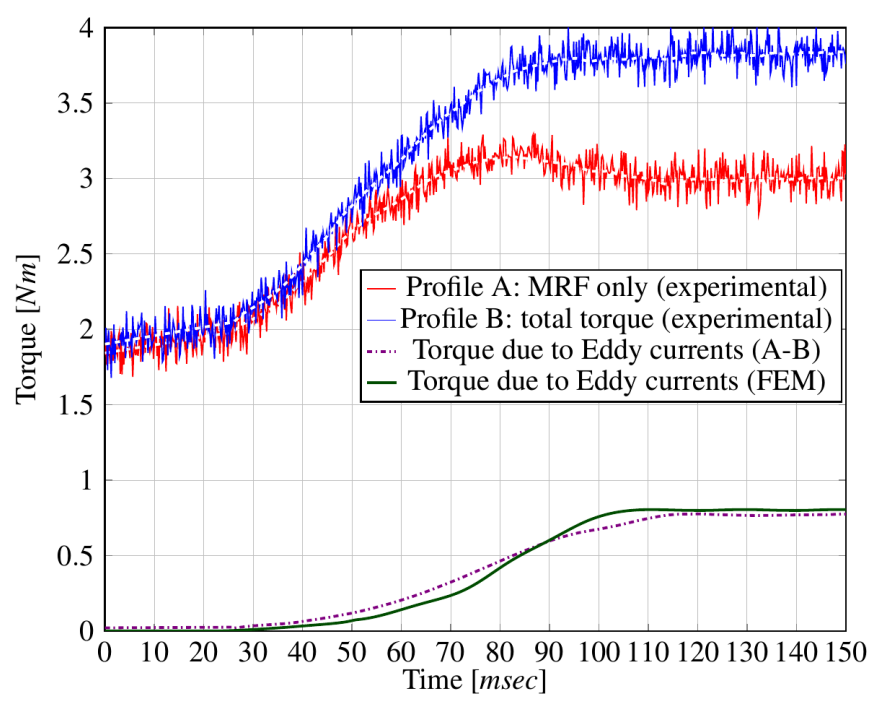

Fig. 13. The torque profiles: simulations and measurements

ascribable to the electrodynamic effects of the eddy currents. Then, exploiting the results of these two experiments, the electromagnetic torque due to the eddy currents only, can be obtained indirectly as a (mathematical) difference between the two measured torques (the dashdotted line in Fig. 13). In the same figure, this latter torque was compared with the simulated electromagnetic torque (see previous section), confirming the ability of the FEM model to predict the behavior of the device also under dynamic conditions. Finally, the results indicate that the total torque was increased by about $27 \%$ as compared with the clutch without the copper sheet.

\section{A. The real clutch operation}

The analysis carried out in the previous sections was performed with a given profile of relative speed $\Delta \Omega$ between the primary and secondary clutch shaft (see Fig. 6), useful in investigating the contribution of the eddy currents. Now, in order to analyze the real behavior of the clutch, let us assume that it is initially in a disengaged condition (OFF state). Under this condition, the fluid is not excited, the eddy currents are negligible and the parasitic torque due to the friction and bearings is not able to actuate the primary shaft; namely, it is at rest $\left(\Omega_{1}=0\right)$, while the secondary one, linked to the engine shaft, rotates at a fixed speed $\left(\Omega_{2} \simeq 1500 \mathrm{rpm}\right)$. When it is necessary to transmit the torque, the spring pushes the $\mathrm{PM}$ in the ON state and, if the torque value is high enough to actuate the load, the primary shaft eventually reaches the same speed as the secondary one and the mechanical slip becomes zero: $\Delta \Omega=\Omega_{2}-\Omega_{1} \simeq 0$. The system attains its mechanical steady state at the end of an electro-mechanical transient whose duration depends on the system characteristics in terms of masses, inertias, spring constant, pneumatic actuator, and so forth. However, when the system is in its mechanical steady condition, the contribution to the torque due to the eddy currents vanishes $(\Delta \Omega=0)$ and the torque transmission is assured only by the MRF.

This is a very important result that allows a smart increase of the clutch transmissible torque. As a matter of fact, during 
the transient phase, while $\Delta \Omega \neq 0$, beside the MRF torque contribution, the system produces also an extra torque due to the eddy currents useful to accelerate the primary shaft; once the system reaches its mechanical equilibrium, since the slip is zero, the eddy currents cannot be induced and the clutch transmits the torque only by means the MR fluid.

The theoretical and experimental analysis described in [2],[17],[12], showed that the transition of the clutch between the disengaged and the engaged condition (that is the PM movement from the OFF state to the ON state) takes about 400 to $500 \mathrm{~ms}$. On the basis of this result, two different analyses are performed. In the first one, the behavior of the clutch when the PM passes from the OFF to the ON state via a number of fixed axial PM positions is investigated. For each PM axial position, the steady state torque is analyzed when the primary shaft is kept blocked and the secondary shaft rotates at a constant speed $\Omega_{2} \simeq 1500 \mathrm{rpm}$. Fig. 14 shows the results of the simulations compared with the experimental data as a function of the PM displacement along its path from the OFF to the $\mathrm{ON}$ state. The results show a good agreement between the numerical simulations and the experimental measurements.

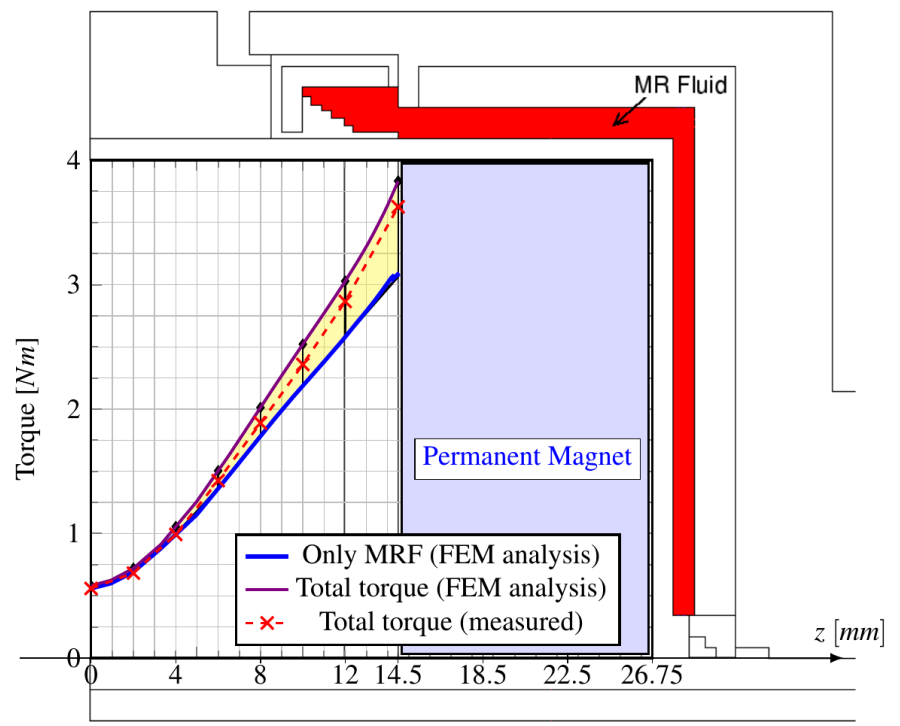

Fig. 14. The torque profile vs PM displacement: simulations and experimental measurements

The second analysis, instead, is aimed at investigating the real dynamic behavior of the clutch. Initially the secondary shaft rotates at constant speed of about $1500 \mathrm{rpm}$, while the PM system is in the OFF position. The primary shaft, loaded with a constant torque of about $2 \mathrm{Nm}$, is at rest due to a low level of transmissible torque (clutch in its disengaged condition). At the time $t=0 \mathrm{~ms}$, the PM system is pushed toward the ON position by the preloaded spring. As a consequence, the primary shaft progressively increases its speed until it reaches the same speed of the secondary shaft, performing the clutch engagement.

Figs. 15(a) and 15(b) show the profile of the experimental torque and primary shaft speed vs time during the transition of the clutch between the disengaged and the engaged condition, produced by the original prototype (red line) and by the new one with the copper sheet (blue line). As for the torque, since in the previously developed device the contribution of the eddy currents is negligible, the difference between the two curves can be mainly ascribable to the electromagnetic torque, obtained by the interaction between the eddy currents in the copper sheet and the magnetic induction.

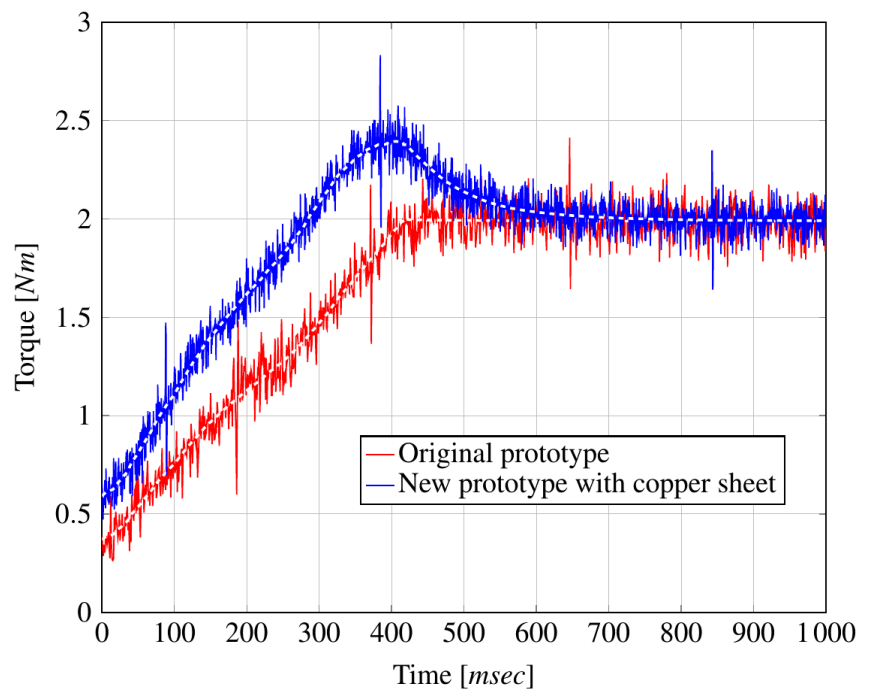

(a) torque

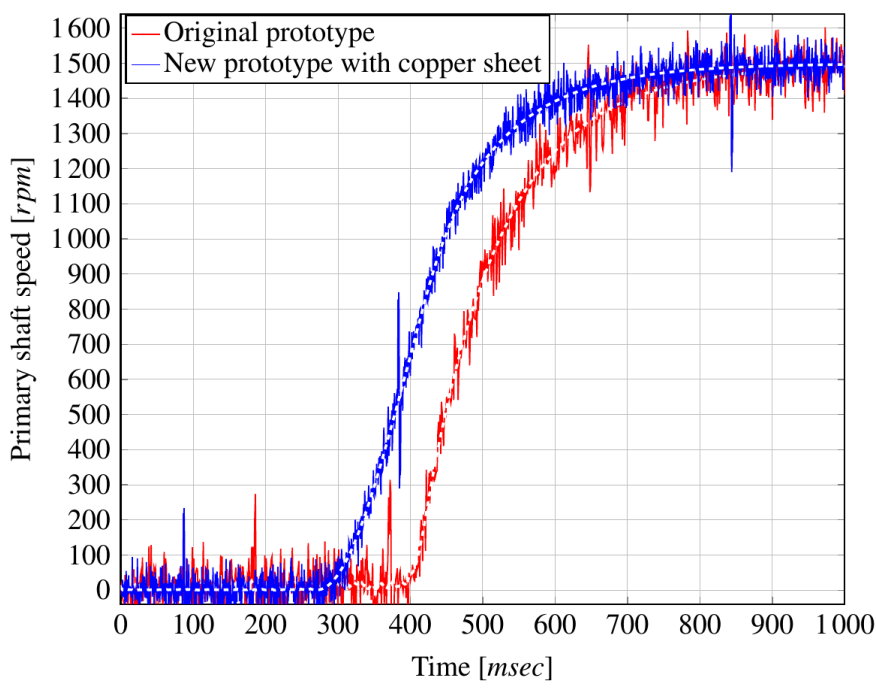

(b) primary shaft speed

Fig. 15. The experimental quantities vs time during the transition of the clutch between the disengaged and the engaged condition

The presence of the eddy currents should be carefully investigated also from a thermal point of view. It is well known that the temperature increasing has negative effects on the torque transmission characteristic of an MRF-based clutch [18], [19]. This problem could be more prominent in the proposed clutch since electrodynamic effects contribute to further increase the temperature of the MR Fluid. Although some very preliminary experimental tests carried out by using a thermographic camera showed that the eddy currents have a relatively low contribution, further investigations about the thermal behavior will be needed. Finally, it is noteworthy to 
highlight that the contribution of the eddy currents to the temperature rising occurs only during the mechanical transient, which is of very short duration (typically $0.5 \mathrm{~s}$ ), for each OFFON cycle.

\section{CONCLUSION}

In this paper an electrodynamic/magnetorheological clutch, activated by using permanent magnets, was described. The interaction between the eddy currents induced in the conductive materials and the magnetic induction produces an electromagnetic torque which can be added to that of the MRF, increasing the performance of the clutch during the engagement phase. Once the two shafts have reached the same speed, the eddy currents (and consequently the electromagnetic torque) vanish and the torque transmission is assured by the MRF only. The device was analyzed using a 3-D finite-elements code, capable of taking into account the B-H functions of the nonlinear materials (e.g. MRF, Permanent Magnets and ferromagnetic materials) and the movement of the different parts. Finally, some experimental measurements were taken on a prototype to validate the FEM model.

\section{ACKNOWLEDGMENT}

This work was funded by Pierburg Pump Technology Italy, S.p.A., within a framework of a project supported by Regione Toscana, P.O.R., C.R.e.O., F.E.S.R. 2007-2013.

\section{REFERENCES}

[1] R. Rizzo, A. Musolino, F. Bucchi, P. Forte, and F. Frendo, "Magnetic fem design and experimental validation of an innovative fail-safe magnetorheological clutch excited by permanent magnets," IEEE Transactions on Energy Conversion, vol. 29, pp. 628-640, Sept 2014.

[2] F. Bucchi, P. Forte, and F. Frendo, "A fail-safe magnetorheological clutch excited by permanent magnets for the disengagement of automotive auxiliaries," Journal of Intelligent Material Systems and Structures, vol. 25, no. 16, pp. 2102-2114, 2014.

[3] H. Bose, T. Gerlach, and J. Ehrlich, "Magnetorheological torque transmission devices with permanent magnets," Journal of Physics: Conference Series, vol. 412, p. 012050, 2013.

[4] G. L. Johnston, W. C. Kruckemeyer, and R. E. Longhouse, "Passive magnetorheological clutch," U.S. Patent, p. 5848678, 1998.

[5] D. Guth, D. Cording, and J. Maas, "Mrf based clutch with integrated electrical drive," in Advanced Intelligent Mechatronics (AIM), 2011 IEEE/ASME International Conference on, pp. 493-498, July 2011.

[6] M. Jackel, J. Kloepfer, M. Matthias, and B. Seipel, "The novel MRFball-clutch design - a MRF-safety-clutch for high torque applications," Journal of Physics: Conference Series, vol. 412, p. 012051, 2013.

[7] EFFE, "Effe v2.00, user manual," Bathwick Electrical Design Ltd, 2009.

[8] G. Armenio, E. Bartalesi, F. Bucchi, A. Ferri, F. Frendo, P. Forte, R. Rizzo, and R. Squarcini, "Mechanical combustion engine driven fluid pump.," EPO Patent, pp. 114251176.2-2423, 2011.

[9] M. Zubieta, S. Eceolaza, M. J. Elejabarrieta, and M. M. Bou-Ali, "Magnetorheological fluids: characterization and modeling of magnetization,' Smart Materials and Structures, vol. 18, no. 9, p. 095019, 2009.

[10] A. G. Olabi and A. Grunwald, "Design and application of magnetorheological fluid," Materials \& Design, vol. 28, no. 10, pp. 2658-2664, 2007.

[11] Lord-Corporation-Ltd, "http://www.lord.com/products-andsolutions/magneto-rheological-(mr)/mr-products.xml.'

[12] E. Bartalesi, F. Bucchi, and R. Squarcini, "Pneumatic brake assistance arrangement," EPO Patent, p. WO2014029444 (A1), 2014.

[13] J. A. Tegopoulos and E. E. Kriezis, Eddy Currents in Linear Conducting Media. Elsevier-Studies in Electrical and Electronic Engineering, 1985.

[14] W. R. Smythe, Static and Dynamic Electricity. McGraw-Hill, 1950.

[15] E. Cardelli, "A general hysteresis operator for the modeling of vector fields," IEEE Transactions on Magnetics, vol. 47, no. 8, p. 20562067, 2011.
[16] A. F. E. Cardelli, E. Della Torre, "A general vector hysteresis operator: Extension to the 3-d case," IEEE Transactions on Magnetics, vol. 46, pp. 3990-4000, December 2010.

[17] F. Bucchi, P. Forte, and F. Frendo, "Experimental characterization of a permanent magnet magnetorheological clutch," ASME 2012 11th Biennial Conference on Engineering Systems Design and Analysis, vol. 4, pp. 345-355, 2012.

[18] S. G. G Yildirim, "Experimental study on heat transfer of the magnetorheological fluids," Smart Materials and Structures, vol. 22, no. 8 , pp. 1-8, 2013.

[19] F. Bucchi, P. Forte, and F. Frendo, "Temperature effect on the torque characteristics of a magnetorheological clutch," Mechanics of Advanced Materials and Structures, vol. 22, no. 1-2, pp. 150-158, 2015. 